\title{
Perinatal and current maternal socio-demographic, health and lifestyle factors influencing children's BMI and waist circumference at 7-year follow-up
}

\author{
C. M. Murrin, D. O’Mahony and C. C. Kelleher For The Lifeways Study Cohort Study Steering Group \\ School of Public Health and Population Science, Woodview House, University College Dublin, Belfield, Dublin 4, \\ Republic of Ireland
}

The prevalence of overweight and obesity in young children cannot be fully explained by current diet and activity patterns. A life-course perspective, including pre- and postnatal familial characteristics, may provide a greater understanding of the current trends.

The Lifeways Cross-Generation Study is a prospective cohort study of Irish children, their parents and grandparents. Pregnant mothers were recruited at their first antenatal visit in 2001 resulting in 1094 infants. At school entry age $63 \%$ of families were followed up in $2007-8$. Socio-demographic, health and lifestyle data were collected using questionnaires completed by the mother during the first trimester of pregnancy (time 1) and at follow-up (time 2). Perinatal data were obtained from maternity hospital records. Height, weight and waist circumference measurements were conducted at follow-up by trained researchers in the family home. Measurements were available for 585 children aged between 4 and 6 years. The UK 1990 growth charts were used to calculate BMI standard deviation scores (SDS), which provide a continuous BMI variable adjusted for age and gender. The outcome measures, BMI SDS and waist circumference and maternal factors from time 1 and time 2, were analysed using $\chi^{2}$, univariate and multiple linear regression statistical methods. Maternal factors included age, region, education, employment, marital status, General Medical Service status, smoking status, alcohol use, oral contraceptive pill, special diets, supplement use, physical activity, parity, hypertension, diabetes and postnatal depression.

Significant variables from $\chi^{2}$ and univariate analysis were used in multiple linear regression analysis. Two models were constructed for each outcome variable ( $a$ and $b$ ) based on maternal predictors at time 1 (including perinatal factors) and at time 2 follow-up. All models were adjusted for maternal age, region, education and marital status. Child age and gender were also adjusted for in the models predicting waist circumference. The first model (1a) showed that during pregnancy mothers who followed a special diet $(\beta-0.159, P=0.01)$ and those who took supplements $(\beta-0.179, P<0.001)$ had an inverse association with their child's BMI SDS. Mothers who suffered from hypertension in pregnancy were more likely to have children with higher BMI $(\beta 0.130, P=0.03)$. None of the maternal factors during pregnancy were predictive of child waist circumference (1b).

In the second model (2a) mothers who were not married were positively associated with BMI SDS ( $\beta$ 0.126, $P=0.02)$. Not taking supplements was, again, significantly associated with increasing BMI $(\beta 0.134, P=0.008)$. Drinking alcohol in a typical week was inversely associated with BMI SDS $(\beta-0.09, P=0.08)$. In model $2 \mathrm{~b}$ increasing waist circumference was significantly associated with mothers who did not take supplements $(\beta 0.105, P=0.06)$ and with mothers who were not married at time $2(\beta 0.152, P=0.01)$. A fully-adjusted model was created for BMI SDS that included the significant variables from the previous two models. The main predictors were hypertension during pregnancy $(\beta 0.132, P=0.01)$ and not taking supplements during pregnancy $(\beta 0.118, P=0.02)$. Use of supplements at time 2 was borderline significant $(\beta 0.085, P=0.09)$.

The present study indicates that maternal health and lifestyle factors in utero exert influences on BMI in early childhood but not waist circumference. However, maternal health and lifestyle behaviours in early childhood influence both BMI and waist circumference. Behavioural and lifestyle interventions aimed at reducing obesity in children should target pregnant women and mothers of young children. 